\title{
Travel time cost functions for urban roads: a case study in Italy
}

\author{
A. Cartenì ${ }^{1} \&$ V. Punzo ${ }^{2}$ \\ ${ }^{I}$ Department of Civil Engineering, University of Salerno, Italy \\ ${ }^{2}$ Department of Transportation Engineering, \\ University of Napoli Federico II, Italy
}

\begin{abstract}
Cost functions are commonly used in traffic assignment to account for the effects of congestion on road link performances. Despite their influence on assignment results, not many effective functions are available at least for urban roads. Indeed, the effects of congestion on the travel time required to cross an urban link have often been considered negligible in comparison with those on delays at intersections. Nonetheless, traffic flow disturbances like side-parking can appreciably affect link travel time with the rise in congestion, especially in the roads of ancient centres in historical cities. Hence this paper presents a travel time function for urban road links including the effect of side-parking, secondary streets and road winding on the worsening of performance due to congestion. Model parameter identification was based both on empirical and experimental data, the latter having been obtained by means of a calibrated micro-simulation model. Cross-validation results do not exclude the possibility of applying the proposed function to different urban areas.
\end{abstract}

Keywords: cost function, travel time.

\section{Background}

Cost functions are commonly used in traffic assignment to account for the effects of congestion on road link performance. In static supply models, the "generalized transport cost" on a link is generally expressed as the weighted sum of three terms: running time, waiting time at intersections and monetary cost. While models for the latter two terms are widespread in the literature, little research in urban contexts has been carried out to define a function between travel time and 
traffic flow (see for example Festa and Nuzzolo [12], for an Italy-based study). Indeed, the effects of congestion on the travel time required to cross an urban link have been often considered negligible in comparison with those on delays at intersections. On the other hand, costs of gathering empirical data do not encourage such studies: for this type of survey, it is necessary to measure the geometrical-functional features of the roads as well as running times of all the vehicles with the varying of traffic conditions. Current employment of computerbased technologies, such as software for identifying car plate numbers, makes data acquisition and processing easier but costs and reliability of such systems still represent restrictive factors.

As stated above, in static supply models (especially for urban applications) the link generalized transport cost is generally expressed as the result of a weighted sum of three terms (see Cascetta [6]):

$$
c_{l}=b_{1} t^{r}{ }_{l}(\boldsymbol{f})+b_{2} t^{w}{ }_{l}(\boldsymbol{f})+b_{3} c m_{l}(\boldsymbol{f})
$$

where $t_{l}{ }_{l}(\boldsymbol{f})$ is the running time on link $l$ as a function of the flow vector $\boldsymbol{f}, t^{w}{ }_{l}(\boldsymbol{f})$ is the waiting time at intersections, $c m_{l}(\boldsymbol{f})$ is the monetary cost and $b_{1}, b_{2}, b_{3}$ are the weights.

For uninterrupted flows, waiting time disappears. In such a case, the best known function is that by the Bureau of Public Roads [5]: $t_{l}^{r}\left(f_{l}\right)=t_{0, l}\left(1+\alpha\left(f_{l} / C_{l}\right)^{\beta}\right)$; in which the travel time $t_{l}^{r}\left(f_{l}\right)$ is a function of the free-flow travel time, $t_{0, l}$, and of the flow-capacity ratio, by way of coefficients $\alpha$ and $\beta$. This type of function has been proposed for urban areas as well. For example, Davidson [9] proposed a single function for estimating both the time components (running and waiting time): $t_{l}\left(f_{l}\right)=t_{0, l}\left(1+\alpha \cdot f_{l}\left(C_{l}-f_{l}\right)\right)$, that is asymptotic for values of $f_{l}$ (flow on link $l$ ) equal to the capacity flow, $C_{l} \alpha$ is a parameter that depends on the features of the road and on environmental constraints as well. The use of asymptotic functions such as Davidson's was subsequently suggested as an instrument which might bind assigned flows to values lower than the link capacities (Daganzo [8]), even though Boyce et al. [4] showed that these functions do not provide realistic travel times on congested links.

Yet in an urban context it is preferable to calculate the link cost as a sum of the three terms of (1). Cost attribute is represented by all the cost items as perceived by the user. In model applications these are very often identified with tolls, where applicable, and with fuel consumption. For waiting time at intersections different functions have been proposed in the literature; among these the best known are the ones by Webster [19], Doherty [10], Akcelik [2] and Highway Capacity Manual [14]. For running time, available functions are not so numerous. Some of these estimate running time starting from link average speed $V_{l}^{\text {running }}$ and link length $L_{l}: t_{l}^{r}\left(f_{l}\right)=L_{l} / V_{l}^{\text {running }}\left(f_{l}\right)$.

A well-known expression of the average speed in stationary flow conditions is that of the Greenshield model [13], based on experimental surveys: $V_{l}^{\text {running }}=V_{0, l} / 2+\sqrt{V_{0, l} \cdot\left(V_{0, l} / 4+f_{l} / k_{j, l}\right)}$; where $V_{0, l}$ is the average free-flow speed and $k_{j, l}$ is the density at capacity. 
Festa and Nuzzolo [12] have proposed a statistical-descriptive function representing the link average running speed as depending on some physical and functional characteristics of road and traffic flow:

$$
V_{l}^{\text {running }}=a_{0}+a_{1} L u_{l}+a_{2} S_{l}+a_{3} W_{l}^{2}+a_{4} H_{l}+a_{5} I_{l}+\left(a_{6}+a_{7} X_{l}\right) \cdot\left(f_{l} / L u_{l}\right)^{2}
$$

where: $V_{l}^{\text {running }}$ is the running speed on link $l[\mathrm{~km} / \mathrm{h}] ; L u_{l}$ is road width (reduced by the space occupied by parked vehicles) [m]; $S_{l}$ is the average road slope [\%]; $W_{l}$ is average road winding $\in[0 ; 1] ; H_{l}$ is the level of circulation hindrance due to pedestrians and parking movements $\in[0,1] ; I_{l}$ is the number of secondary intersections per kilometre; $X_{l}$ is a dummy variable equal to 1 if the road does not allow overtaking, zero otherwise; $f_{l}$ is the link flow in [vehicles $/ \mathrm{h}$ ].

However, due to the shortage of data available for the calibration, the model by Festa and Nuzzolo is significant in a limited range of flows (as recognized by the authors themselves).

Following their work this paper proposes a new specification of an urban cost function especially suited for historical centres of old cities. It was calibrated on a large database of empirical data expanded with experimental data obtained from a properly calibrated and validated traffic microscopic simulation model. Currently, traffic micro-simulation models are increasingly being employed as "virtual laboratories" to carry out experiments that cannot easily be performed in the real world (see e.g. Barcelò et al. [3]; Yang et al. [20]).

The paper is organized in three sections; in the first the calibration methodologies (including the calibration and validation of the microscopic simulator) are described; in section two, calibration results are reported and finally research conclusions are discussed.

\section{Calibration methodology}

As mentioned above, the aim of this work is to estimate a mathematical function to provide the running time in an urban context depending on geometric and functional features of roads and on traffic conditions (traffic flows). This basically means specifying and calibrating a model: $t_{l}^{r}=g\left(f_{l}, x_{l}, x_{2} \ldots x_{n}\right)$ where $t_{l}^{r}$ is the running time on link $l, f_{l}$ is the traffic flow on link $l$ (we assumed that traffic flow is not influenced by flows in the opposite direction) and $x_{1}, x_{2} \ldots x_{n}$ are some road attributes influencing running speed.

As the available data referred to road sections far away from the intersections it was necessary to subdivide calibration into two steps. In the first step, running time was calibrated on these data without considering time losses due to acceleration and deceleration phases. In the second, an adjusting term $c\left(L_{l}\right)$, depending on the length of the infrastructure $L_{l}$, was added as a result of kinematic considerations, such that:

$$
t_{l}^{r}=t_{l}^{*} \cdot c\left(L_{l}\right)=\left(L_{l} / V_{l}^{\text {running }}\right) \cdot c\left(L_{l}\right)
$$

where $t_{l}^{*}$ is the running time of road $l$ in "ideal" conditions (i.e. neglecting acceleration and deceleration lost times) and $V_{l}{ }^{r}$ is the running speed over road $l$.

To estimate the model parameters the least squares method was used. In order to generate a significant sample a method was set up which integrates 
conventional and modern survey techniques. Combined with conventional research, a number of surveys were carried out by means of GPS equipment on the one hand while, on the other, experiments were conducted on a microsimulation model, used as a "virtual test field", which was duly calibrated and validated.

Conventional surveys consisted in measuring relevant geometric quantities of a number of infrastructures, along a predetermined period of time, together with the travel times of single vehicles between two sections chosen to be measured, in different conditions of vehicle flow. GPS surveys were used to calibrate some significant parameters of the micro-simulation model (in particular, the desired speed) as well as to provide detailed speed profiles between the measuring sections on a preliminary check of surveyed data. The micro-simulation model was therefore calibrated, validated and used to integrate survey data, as described below in greater detail.

\subsection{Road surveys}

Surveys were carried out along 17 roads in the city of Naples (Italy) under different traffic conditions. To measure travel times the moments when all vehicles passed along two road sections were video-recorded. The sections were located as far away as possible from the intersections so that vehicles would not be affected by the influence of the latter (e.g. queue spillback, etc.).

Table 1: $\quad$ Physical road characteristics monitored.

\begin{tabular}{|l|l|c|c|c|c|c|c|c|}
\hline$I d$. & \multicolumn{1}{|c|}{ Road name } & $L$ & $L u$ & $S$ & $W$ & $D$ & $S P$ & $P v$ \\
\hline 1 & Parco Margherita & 532 & 4.0 & 0 & 0.66 & 0.66 & 1 & 0 \\
\hline 2 & Cintia & 302 & 6.0 & 0 & 0 & 0.66 & 1 & 1 \\
\hline 3 & Orazio (downhill) & 653 & 3.5 & 0 & 0.66 & 0.33 & 0.2 & 1 \\
\hline 4 & Orazio (uphill) & 653 & 3.5 & 5 & 0.66 & 0.33 & 0.2 & 1 \\
\hline 5 & Posillipo (direction 1) & 243 & 4.4 & 0 & 0.33 & 0 & 0.15 & 0 \\
\hline 6 & Posillipo (direction 2) & 243 & 4.4 & 0 & 0.33 & 0 & 0.15 & 0 \\
\hline 7 & Pozzuoli (direction 1) & 402 & 2.6 & 0 & 0 & 0.66 & 1 & 1 \\
\hline 8 & Pozzuoli (direction 2) & 402 & 2.6 & 0 & 0 & 0.66 & 1 & 1 \\
\hline 9 & M.C.Savoia & 338 & 3.5 & 0 & 1 & 0.33 & 1 & 0 \\
\hline 10 & Michelangelo (direction 1) & 267 & 4.2 & 0 & 0.33 & 0.33 & 0.8 & 0 \\
\hline 11 & Michelangelo (direction 2) & 267 & 4.2 & 0 & 0.33 & 0.33 & 0.8 & 0 \\
\hline 12 & Caravaggio (direction 1) & 265 & 3.3 & 0 & 0.33 & 0.33 & 1 & 1 \\
\hline 13 & Caravaggio (direction 2 & 265 & 3.3 & 7 & 0.33 & 0.33 & 1 & 1 \\
\hline 14 & Tasso (direction 1) & 165 & 3.8 & 0 & 0.66 & 0 & 0.5 & 0 \\
\hline 15 & Tasso (direction 2 & 165 & 3.8 & 5.5 & 0.66 & 0 & 0.5 & 0 \\
\hline 16 & Terracina & 339 & 4.0 & 0 & 0 & 0.33 & 0.15 & 1 \\
\hline 17 & Beccadelli & 359 & 8.4 & 0 & 0 & 0 & 0 & 1 \\
\hline \hline \multicolumn{2}{|l|}{ Mean } & 345 & 4.1 & 1.0 & 0.4 & 0.3 & 0.6 & 0.5 \\
\hline Standard deviation & 147 & 1.4 & 2.3 & 0.3 & 0.2 & 0.4 & 0.5 \\
\hline
\end{tabular}


Choice of the infrastructure was determined with respect to road geometry, gradient, bendiness and distress (see table 1) so that a sample as inhomogeneous as possible might be obtained. In particular, each infrastructure was classified according to length ( $L$ in metres), width ( $L u$ in metres), slope ( $S$ in \%), bendiness $(W)$, distress index $(D)$, percentage of side parking $(S P)$ and kind of paving $(P v$, asphalt $=1$, other $=0$ ). As regards bendiness, four levels were identified (see also Festa and Nuzzolo [12]): no bending (which means that the road is straight), scarce bending, average bending and high bending (when means the curve radius is lower than $30 \mathrm{~m}$, thus not allowing speeds higher than $25-30 \mathrm{~km} / \mathrm{h}$ for safety's sake). By the distress index we refer to factors external to traffic affecting vehicle flows, such as bus stops, pedestrians crossing the roadway, side access to dwellings and whatever hinders circulation excluding parked vehicles.

In the estimate of the model proposed, the four road-bending and distress levels have been attributed the values of $0.00-0.33-0.66-1.00$. Each road was monitored for a period of time ranging from one hour at least to two hours at most, split into several observation periods during daytime (peak-hour intervals, average and moderate flows).

\subsection{Calibration and validation of the virtual test field}

Having taken road survey difficulties into account, a traffic micro-simulation model was used as a virtual test field, for the purpose a) of expanding data actually surveyed on the road - by simulating running times relative to unsurveyed flow rate values- and (b) of carrying out virtual surveys along roads differing (in their attributes) from those surveyed. For this purpose, the simulation model chosen, AIMSUN NG (AIMSUN [1]), was calibrated on a set of data relative to four different infrastructures and was then validated on a holdout sample including surveys of four more roads.

As is well-known, a model-based approach (i.e. based on using the model itself for estimating the parameters) consists in comparing model output with the data of the real system, inputs being equal. In the case of a traffic microsimulation model, this procedure has two main criticalities: the impossibility of having the same inputs both for the system and the model, and the huge number of parameters involved (see e.g. Ciuffo et al. [7]). The main input of a traffic simulation model consists of origin-destination (O-D) flows, which can hardly ever be directly observed. This implies that the same flows are subject to the calibration/estimation procedure (often carried out by minimizing the distance between the time series of simulated flows or speeds on a sensor and the observed ones). There is no need here to stress how complex such a procedure is, requiring the resolution of many fixed point problems. O-D flow estimation, for example, requires knowledge of the assignment matrix, which is function of equilibrium running times, which in turn depend on O-D flows (see e.g. Toledo et al. [18]).

The other criticality is represented by the huge number of parameters involved: parameters of route choice and parameters of driving behaviour, whose mutual effect on the simulation output cannot be detached, as already indicated above. 
In this case study, however, the calibration phase did not prove difficult. It consisted in calibrating the model parameters on single road sections, far from the intersections. Hence the level of input demand was exactly known, corresponding to the arrivals reported in the initial counting sections of the roads surveyed. The only parameters to be calibrated were those of the driving models, since no route choice was considered and a test of model performance was not required at special points such as inflows or intersections. The parameters calibrated were (see AIMSUN [1]): section speed limit; percentage of compliance with speed limits; drivers' desired speed; drivers' maximum desired acceleration; drivers' maximum desired deceleration; percentage of overtake (index of propensity to overtake); reaction time (equal among all drivers and coincident with the simulation step).

The measure of performance used for calibration was average running time taken by vehicles to cross the entire section. The choice of this parameter was determined by both the type of application (the model was required to reproduce running time as accurately as possible) and the fact that it represents the aggregate measurement which best captures the effect of driving parameters onto the flow dynamics (indeed, it is directly bound to space mean speed).

As the simulation is stochastic, output measures were obtained by determining the average over 15 replications at each iteration (see Law and Kelton [17], on the "replication/deletion approach" for calculating the number of simulations required to obtain an estimate of the sampling average with a fixed standard of reliability).

By simulating the flows of demand levels surveyed on the roads, the above parameters were calibrated by minimizing the distance between the average of the observed travel times and that of simulated ones using the RMSE statistic.

Special attention was devoted to estimating the desired speed (free-flow speed), which was obtained by employing data in free-flow traffic conditions by means of a probe vehicle equipped with a kinematic differential GPS. By establishing that the speed limits of the basic sections of the supply model were equal to the speeds surveyed and by subsequently calibrating the percentage of acceptance of these limits, each vehicle proved to have variable desired speeds along the road. This is a basic element to consider on simulating the effect of bendiness (see AIMSUN [1], on the acceleration model used).

The model was statistically validated in two subsequent steps. First, model outputs were compared with the data surveyed along the four roads concerned in order to check how fit the model was to adequately represent real conditions. Secondly, the model outputs were compared with the data of a hold-out sample of four roads. The model was checked for its capacity to capture the impact of geometric-functional features of infrastructures on road flow, so as to adequately represent also sections with different features as those for which it had been calibrated ("transferability" of the model to different roads).

In both steps of the validation process, for each road, a paired- $t$ confidence interval (see Law and Kelton [17]) was calculated for the two time series (i.e. empirical and simulated ones) of the average running time of vehicles over a (stationary) period. In other words, the outputs of simulations and surveyed data 
were checked for being independent deviates from identical distributions. Thus, for each of the eight roads surveyed, the model proposed was calibrated both with data as surveyed and with those simulated, and $F$ tests were effected on the parameters of coupled models (a model from survey data and a model from simulated data), to check their actual similarity. The second procedure was in some cases required due to the limited number of data by which the test of the confidence interval might be carried out. After all, such an approach to the comparison (with system meta-models) was especially appropriate, if we consider that the purpose of this study consists in the calibration of a metamodel. It is important to stress here that not all the comparisons were successful. It was surprising to see that validation tests were not successful along roads with a distress index over 0.33 . For a more exact interpretation, more in-depth analysis would be required. Accordingly, additional road surveys were carried out along these infrastructures.

\subsection{Assumption of intra-period stationarity}

The performance function to be implemented forms part of mathematical models assuming intra-period stationarity which means assuming that supply and demand stay constant for long periods so as to allow the system to reach a condition of steady state.

Once surveys were carried out the minimum interval for which assumed intra-period stationarity might be applied was identified. We used the test proposed by Ferrari et al. [11], based on the calculation of the correlation coefficient between the sequences of counts and that of integer numbers, where $n$ is the number of sub-intervals of equal $\theta$ length (assumed as equal to 1 minute, Ferrari et al. [11]) into which the interval $T$ to be tested was divided, and $y_{1}$, $y_{2} \ldots y_{n}$ is the sequence of counts for each of them. It was ascertained whether the mean is steady by testing whether the sequence $y_{1}, y_{2} \ldots y_{n}$ is independent from the one of the first $n$ integer numbers (Kendall and Stuart [16]).

\subsection{Data gathering results}

The sample surveyed consists of 17 roads and about 25,000 measurements. In order to let every road "weigh" in the same way within the regression, survey data were expanded by means of AIMSUN, as stated above, so as to obtain, for each infrastructure, the same number of measurements (about 1,500 observations against the 530 used by Festa and Nuzzolo [12] on average per infrastructure).

\section{Calibration results}

From the data monitored it emerges that running time in an urban context depends on the geometric and functional features of roads. It was also observed that the vehicle flow influence is less marked on the infrastructures with a high bendiness and distress index. Starting from such considerations, for the specification of the term $t_{l}{ }^{*}$ (see equation (3)) the following model was proposed: 


$$
t_{l}^{*}=\frac{L_{l}}{\beta_{0}+\beta_{1} L u_{l}+\beta_{2} S_{l}+\beta_{3} W_{l}+\beta_{4} D_{l}+\beta_{5} S P_{l}+\beta_{6} P v_{l}+\frac{\beta_{7} \cdot\left(f_{l} / L u_{l}\right)^{2}}{1+W_{l}+S P_{l}+D_{l}}}
$$

where: $t_{l}{ }^{*}$ is the running time of road 1 in "ideal" conditions expressed in hours; $L_{l}$ is the road length in $\mathrm{km} ; f_{l}$ is the link flow in veh/h; $L u_{l}$ is the road width in metres; $S_{l}$ is the average road slope in $\% ; W_{l}$ is average road bendiness in values in the interval $[0,1] ; D_{l}$ is the distress index in values in the interval $[0,1] ; S P_{l}$ is the percentage of the road which has side parking; $P v_{l}$ is a kind of pavement dummy variable (asphalt $=1$, other $=0$ ).

Table 2 shows values of calibrated parameters, Student's t statistics, the

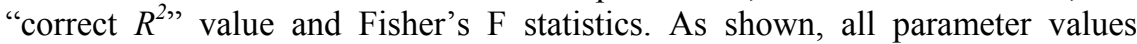
appear to be correct and significantly different from zero. The model (4) was validated by a hold-out sample (cross-validation), consisting of data not used for the calibration procedure. Validation provided good results; the differences among observed running times and the corresponding model value were always less than $15 \%$.

Fig.1 shows the performance of the model (4) for one of the infrastructures concerned with respect to vehicle flow variation.

Table 2: $\quad$ Calibration results.

\begin{tabular}{|c|c|c|c|c|c|c|c|c|}
\hline & $\begin{array}{c}\beta_{0} \\
{[\mathrm{Km} / \mathrm{h}]}\end{array}$ & $\begin{array}{c}\beta_{1} \\
{[\mathrm{Km} / \mathrm{h} \cdot \mathrm{m}]}\end{array}$ & $\left.\begin{array}{c}\beta_{2} \\
{[\mathrm{Km} / \mathrm{h}]}\end{array}\right]$ & $\begin{array}{c}\beta_{3} \\
{[\mathrm{Km} / \mathrm{h}]}\end{array}$ & $\begin{array}{c}\beta_{4} \\
{[K m / h]}\end{array}$ & $\begin{array}{c}\beta_{5} \\
{[K m / h]}\end{array}$ & $\begin{array}{c}\beta_{6} \\
{[K m / h]}\end{array}$ & $\begin{array}{c}\beta_{7} \\
\left.\left[K m \cdot h \cdot m^{2}\right) / v e h i c\right]\end{array}$ \\
\hline value & 29.915 & 3.598 & -0.586 & -13.865 & -10.814 & -6.383 & 4.739 & $-1.052 \mathrm{E}-04$ \\
\hline $\begin{array}{c}t\left(\beta_{i}\right) \\
\left(t_{0.01}= \pm 2.58\right)\end{array}$ & 127.86 & 113.73 & -31.68 & -78.89 & -39.88 & -40.26 & 45.02 & -60.15 \\
\hline & $R_{c o l}^{2}$ & 0.743 & & & $F\left(F_{0.01}\right.$ & $=3.02)$ & 12.545 & \\
\hline
\end{tabular}

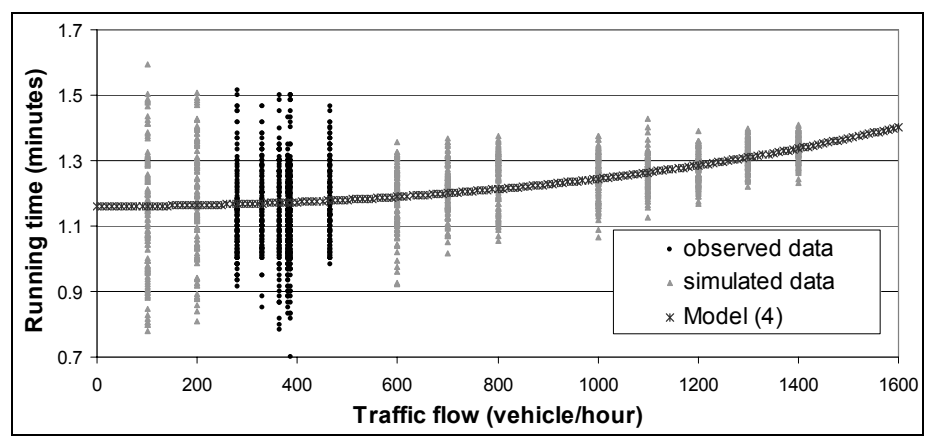

Figure 1: $\quad$ Model (4).

Once term $L_{l} / V_{l}{ }^{r}$ had been estimated, the $c\left(L_{l}\right)$ adjusting term (see equation (3)) was determined. The specification providing the best results was as follows:

$$
c\left(L_{l}\right)=1 /\left(1-\exp \left(-0.472-0.482 \cdot 10^{-2} \cdot L_{l}\right)\right)
$$


By representing this corrective coefficient $c\left(L_{l}\right)$ on a graph its influence may be observed to decrease rapidly as the link length rises and become negligible for lengths exceeding $500 \mathrm{~m}$.

The model (3) was validated by a hold-out sample. This sample consisted of a database of six roads not used for the calibration procedure; the gap between running times surveyed and the values predicted by the model is almost always lower than 15\% (with an absolute mean difference of $9 \%$ and a standard deviation of $5 \%$ ).

To analyze the transferability of the model proposed in the Italian urban context, a comparison was carried out between model (3) and model (2) calibrated by Festa and Nuzzolo [12] (appropriately converted into running times according to the length of the infrastructure). In particular, as regards the sample of roads (nine infrastructures) surveyed by Festa and Nuzzolo [12], model (3) running times and model (2) running time were compared with the values measured by Festa and Nuzzolo [12]. While both models are able to estimate the free-flow running time (percentage differences below 11\%), when flow increases, only model (3) is capable of reproducing the running time measured (model (3) average percentage variation $=13 \%$; model $(2)$ average percentage variation $=140 \%$ ).

In the left-hand part of figure 2 the differences between model (3) and model (2) according to flow variation are represented (concerning one of the Naples infrastructures surveyed, representative of an average condition). It is worth stressing that the model proposed (3) can adequately reproduce the running time surveyed.

To test the transferability of the model to other urban contexts in Italy, model (3) was compared with model (2) re-calibrated by Ignaccolo and Giunta [15] and concerning Catania (a city in southern Italy). The right part of figure 2 shows how model (3) can reproduce the running time quite accurately according to flow variation.
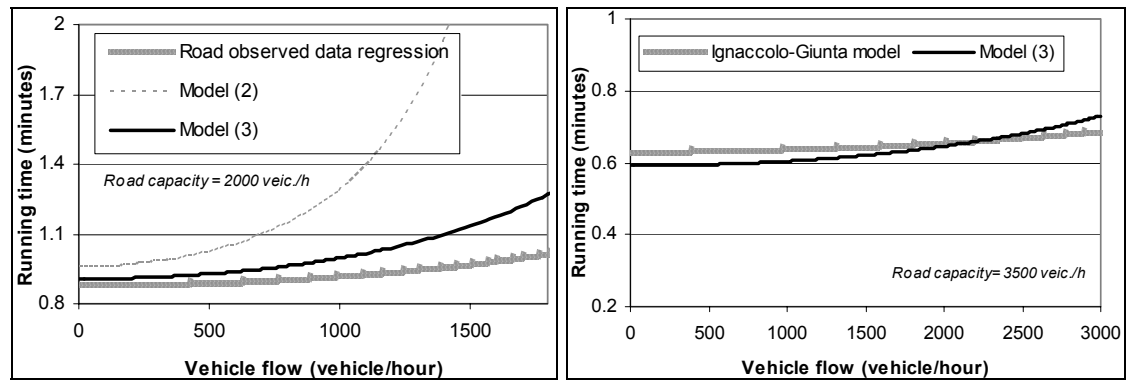

Figure 2: $\quad$ Comparison between model (3) and model (2).

\section{Conclusions}

Scarce availability of running time functions in the literature is probably due to the operating difficulties of carrying out experimental surveys. In this work, the integration of empirical data with experimental ones (from a traffic micro- 
simulation model) allowed us to reduce costs of data acquisition. Therefore, a model was calibrated which might allow estimation of running time as a function of traffic flow. The cost function is especially suited to urban road links of ancient centres in historical Italian cities.

Calibration and validation statistics revealed quite a good fit to the real data of the proposed model, as well as good transferability.

\section{References}

[1] AIMSUN 5.0, Microsimulator User's Manual 5.0.6. Transport Simulation Systems 1997-2005, November 2005.

[2] Akcelik, R., The Highway Capacity Manual delay formula for signalized intersections, ITE Journal, pp. 23-27, 1988.

[3] Barceló, J., Codina, E., Casas, J., Ferrer, J.L. and García, D., Microscopic Traffic Simulation: A Tool For The Design, Analysis And Evaluation Of Intelligent Transport Systems, Journal of Intelligent and Robotic Systems, Theory and Applications, Volume 41, No.2-3, pp.173-203(31), January 2005.

[4] Boyce, D.E., Janson, B.N. and Eash, R.W., The Effect on Equilibrium Trip Assignment of Different Link Congestion Functions, Transportation Research, 15A, pp. 223-232, 1981.

[5] Bureau of Public Roads, Traffic Assignment Manual, U.S. Department of Commerce, Washington DC, 1964.

[6] Cascetta, E., Transportation systems engineering: theory and methods. Kluwer Academic Publishers, 2001.

[7] Ciuffo, B., Punzo, V., Torrieri, V., Framework for calibrating microscopic traffic simulation models, Proceedings of the $86^{\text {th }}$ TRB Annual Meeting, Washington DC, 2007.

[8] Daganzo, C.F., On the Traffic Assignment Problem with Flow Dependent Costs - I and II, Transportation Research, 11, pp. 433-441, 1977.

[9] Davidson, K.B., A Flow Travel Time Relationship for Use in Transportation Planning, Proceedings Australian Road Research Board 3, pp.183-194, 1966.

[10] Doherty, A.R., A comprehensive junction delay formula, LTR 1Working Paper, Department of Transport, 1977.

[11] Ferrari, P., Treglia, P., Cascetta, E., Nuzzolo, A., Olivotto, P., A new method for measuring the quality of circulation on motorways, Transportation Research Vol. 16, No. 5 pp. 399-418, 1982.

[12] Festa, D. C., Nuzzolo, A. (1990), Analisi sperimentale delle relazioni velocità-flusso per le strade urbane, Le Strade, XCII, no. 1266, May 1990.

[13] Greenshields, B., A Study of Traffic Capacity, Proceedings of the Highway Research Board, Vol. 14, TRB, National Research Council, Washington DC, 1934.

[14] Highway Capacity Manual, Transportation Research Board, National Research Council, Special Report 209, 3rd edition. Washington DC, 1994. 
[15] Ignaccolo, M., Giunta, U., Determinazione della curva di deflusso per strade di scorrimento in ambito urbano: rilievi e considerazioni sulla Circonvallazione di Catania, I piani urbani del traffico, edited by de Luca M., Astarita V. , Franco Angeli, 1998.

[16] Kendall, M.G., Stuart, A., The advanced theory of statistics, Vol. III C. Griggin and Co., London, 1968.

[17] Law, A.M., Kelton, W.D., Simulation Modeling and Analysis, 3rd Edition, McGraw-Hill, New York, 2000.

[18] Toledo, T., Koutsopoulos, H.N., Davol, A., Ben-Akiva, M.E., Burghout, W., Andréasson, I., Johansson, T. and Lundin, C., Calibration and Validation of Microscopic Traffic Simulation Tools: Stockholm Case Study. Transportation Research Records 1831, 2003.

[19] Webster, F.W., Traffic Signal Settings, Transp Road Res. Lab. Technical Paper N. 39, 1958.

[20] Yang, Q., Koutsopoulos, H.N. and Ben-Akiva, M.E., A Simulation Laboratory for Evaluating Dynamic Traffic Management Systems, Transportation Research Record, 1710, pp. 122-130, 2000. 